Testogan), die aus Hoden, Prostaladrüsen und Ovarien von Tieren hergestellt werden.

Wichtiger sind die Mutterkornpräparate, obwohl sie auch nicht annähernd die Bedeutung baben wie in der Humanmedizin, wo sie als Uterina in Gynäkologie bei der Geburtshilfe als wehenanregende und besonders als blutstillende Mittel bei postpa:talen Uterushlutungen und bei den Menstruationsanomalien unentbehrlich sind. Da bei den Haustieren ron einer Menstruation, in der Art und Weise, wie sie bei Frauen auftritt, kaum gesprochen werden kann, ist die therapeutische Anwendung der Secalepräparate nur bei Schwergeburten indiziert, die auf unzureichende Wehentïitigkeit und Erschlaffung der Uterusmuskulatur zurückzufïhren ist, und zwecks schnellerer Ausstoßung der zuriickgebliebenen Nachgeburt. Zur Herbeiführung des künstlichen Abortus sind meist toxische Dosen nötig, weshalb die Verwendung zu diesem $\mathrm{Z}$ wecke gefährlich ist.

Iber den chenischen Aufbau und die physiologischen Eigenschaften der wirksamen Bestandteile der Secale cornutum war man lange im unklaren, und erst die wissenschaftlichen Arbeiten der letzten Zeit haben Wandel geschaffen. Neben einer ganzen Anzahl von physiologiseh unwirksamen Körpern sind die Alkaloide Ergotinin und Hydroergotinin (Frgotoxin) als aktive Substanzen isoliert worden, deren chemische Struktur aber noch nicht genlugend geklärt ist. Dagegen sind die aus wasserigen Extrakten gewonnenen aromatischen Aminobasen als Phenyl-,p-OxyphenyJ-, $\beta$-Imidazolyl-und $\beta$-Indoly athylamine erkannt worden. - Ganz ähnlich wie Secale wirkt Hydrastis canadensis, sowie das daraus hergestellte Alkaloid Hydrastin, dessen Spaltungsprodukt Hydrastinin und das Cotarn in -- ein Methoxyhydrastinin-, gewonnen durch Spaltung des Opiumalkaloids Narcotin. Als Handelspräparate sind bekannt geworden das salzsaure und plithalsaure Salz des Cotarnins (Sty pticin und Styp tol). Auch das Suprarenin und die Hypophysenpraparate (Hypophysin und Pituitrin) haben neuerdings in der Menschenmedizin den Mutterkornpräparaten erfolgreich Konkurrenz gemacht. In der Veterinärmedizin werden fast ausschlieblich die Mutferkorndroge und die daraus hergestellten offizinellen Extrakte angewendet. [A. 35.]

\section{Auch ein Wort zum Chemieunterricht an den höheren Schulen.}

Von Oberstudienrat Dr. KǓsPERT, Nürnberg. (Eingeg. 9.:3. 1922.)

Für den Unterricht in der Chemie, wie iberhaupt, ist es eine grundsătzliche, die Geister scheidende Frage, ob die höhere Schule anzusehen ist als eine "Kenntnisübermittlungsschule" oder als eine Erkenntnisbildungsschule

Die Früchte eines bloß mitteilenden und darum Kenntnisse bäufenden Lehrverfabrens wird man mit großer Wahrscheinlichkeit immer vermuten dürfen, wo der Hochsebullehrer über mangelnden Fleiß3 klagt gerade derjenigen, die vorher schon einen Lehrgang der Chemie durchgemarht haben. Schon aus diesem Grunde, noch mehr aber deshalb, weil wir die Chemie als ein Denk- und nicht als ein Lernfach eingeschätzt wissen wollen, muß das Ziel heißen: Erkenntnisbildungsschule!

Nur ein ganz langsames Aufbauen der Begriffe, die Begriffsbildung auf dem heuristischen Weg aus den vorangestellten (nicht zum Beweis für aufgestelite Thesen nachträglich gebrachten uad mit Formeln „erläuterten“) Tatsachen schafft UUbung im logischen Denken. Dieser Weg schaltet ganz automatisch Wissensbreite aus und nimmt so der Hochschule nichts vorweg. Denn er wird nur zu den Grundvorstellungen führen und wird diese keinesfalls in ihrer letzten und feinsten Ausprägung erstreben; er bietet Erkenntnis vorformen, ausreichend für jene Schüler, die sich später nicht den Naturwissenschaften zuwenden, und doch auch so beschaffen, daß sie den künftigen Chemiker durstig genug sein las-en auf die hohe Schule.

Wer für diese Auffassung eintritt, muB sich freilich gefallen lassen, daß man ihm mangelnde Tiefe vorwirft, und wer da versucht, einen Lehrgang zu geben, in welchem der methodisch langsamen Begriffsentwicklung gedient sein soll, dem hält man unnötige Stoffmasse vor. Statt dessen wird ein Lehrgang verlangt, der die lebendige Bedeutung der Chemie ins rechte Licht rückt. Gat! Aber taucht da nicht wieder die Gefahr der Kenntnisübermittlungsschule auf? Die Gefahr, daß Schilderung zur Hauptsache, Einsichtgewinnen vom Versuch aus zur Nebensache wird? Der Schulchemiker wird gewiß nicht unterlassen, technische Betriebe mit seinen Schülern zu besuchen, Licht- und Laufbild auszunützen und hierdurch die Vorstellungen von der wirtschaftlichen Bedeutung der Chemie lebendig machen, er weiß aber auch, daß er Erkenninisbildner sein muB, wenn er in seinem Fach an der allgemeinen Geistesbildung mitbauen will. Versuche, die Schulbücher auf diese Richtung einzustellen und dadurch die Formelkrämerei zu beseitigen, sollte man aus den vorgetragenen Gründen nicht zurückweisen; auch dann nicht, wenn der einzelne Wurf als mißglürkkt empfunden wird, weil darin der methodische Zeitaufwand als Druckzeilenhäufung, der Kompromiß mit vorbandenen Vorschriften als Stoffülle, die Rücksicht auf die formale Einprägbarkeit der gewonnenen Erkenntnisse als Trockenheit der Darstellung erscheint. Dies zugleich meine Erwiderung auf die Kritik von Alfred Stock in dieser Zeitschrift (35, 35 [1922]).
Einige Bemerkungen $z u$ den vorstehenden Ausführungen.

Von AlFRED STOCK.

Die Schriftleitung war so liebenswürdig, mir die Außerungen des Herrn Oberstudienrats Dr. Küspert vor der Drucklegung zuzustellen. Ich benutze gern die Gelegenheit, meinerseits einige Sätze daranzuknubpen, damit nicht Leser, welche meine Ansichten und Veröffentlichungen über den Schulchemieunterricht nicht kennen, ein falsches Bild von meiner Auffassung bekommen.

Meines Erachtens soll die höhere Schule zugleich „Frkenntnisschule $^{*}$ and "Kenntnisschule" sein. Sie soll zum Beobachten, zum eigenen Denken und zum logischen Verknüpfen des Neuen mit dem Bekannten erziehen. Aber sie soll den Schülern auch die Kenntnisse verschaffen, welche sie im praktischen Leber brauchen. Gerade der Chemieunterricht kann die Synthese von "Erkenntnisbildung" wind "Kenntnisübermittlung " aufs gl jucklichste vollziehen. Es ist keineswegs nötig, daß sich die Geister mit den Schlachtrafen „Kenntnisübermittlungsschule" und "Erkenntnisbildungsschule" scheiden.

$\mathrm{Daß}$ die "Gefahr der Kenntnisübermittlungsschule" auftauchen muß, wenn man die Iebendige Bedeutung der Cherie beim Schulunterricht ins richtige Licht rückt, will mir nicht in den Sinn. Die Erkenntnisbildung braucht nicht zu leiden, wo man sie an praktisch wichtigen Dingen stählt. Im Gegenteil: Sie gewinnt an Wert für das Leben. Non scholae, sed vitae discimus!

[A. 70.]

\section{Verein deutscher Chemiker.}

\section{Hauptversammlung zu Hamburg} 7. - 10. Juni 1922.

Die diesjährige Hauptversammlung findet in Hamburg vom 7. bis 10. Juni statt. Wenn es auch dem Ortsausschuls mit Rücksicht auf die schwierigen wirtschaftlichen Verhältnisse nicht möglich sein wird, die Versammlung äutterlich so glanzvoll $\mathrm{zu}$ gestalten, wie es in manchem Vorjuhre der Fall war, so ist er dennoch bemüht, den Fachgenossen und Gästen die Tage, die sie in Hamburg zubringen werden, anregend und angenehm zu gestalten. Wie in früheren Jabren findet eine große Zahl wissenschaftlicher Vorträge statt. Hiesige industrielle Werke und eine Werft haben zur Besichtigung ihrer Anlagen eingeladen. Die Ausstellung von Maschinen, Apparaten und Hilfsmitteln für chemische Industrie und Forsebung (Achema), die in der Zeitschrift bereits vor einigen Wochen den Grundriß ibrer Räume veröffentlicht hat (Heft 12, S. VII-X), wird viele interessante Neuheiten bieten.

Ein größerer Ausflug muß in diesem Jahre unterbleiben; aber eine gemeinsame Fahrt auf der Elbe wird dafür Entschädigung bieten.

Das Damenprogramm ist reichhaltig gestaltet und sieht unter anderem einen Ausflug narb Friedrichsrub vor.

Das genaue Programm wird in 8 Tagen veröffentlicht und gleichzeitig der Zeitschrift der Bestellschein für die Teilnehmerkarte beigelegt. Es empfiehlt sich alsdann, möglichst sogleich die Anmeldung zu vollziehen, damit der Ortsausschul in der Lage ist, die sehr schwierige Frage der Unterkun ftsbeschaffung zu allseitiger Zufriedenheit z11 lösen. Ortsausschuß für die Hauptversammlung 1922. Gescbäftsstelle, Hamburg 36, Jungiusstr. 9.

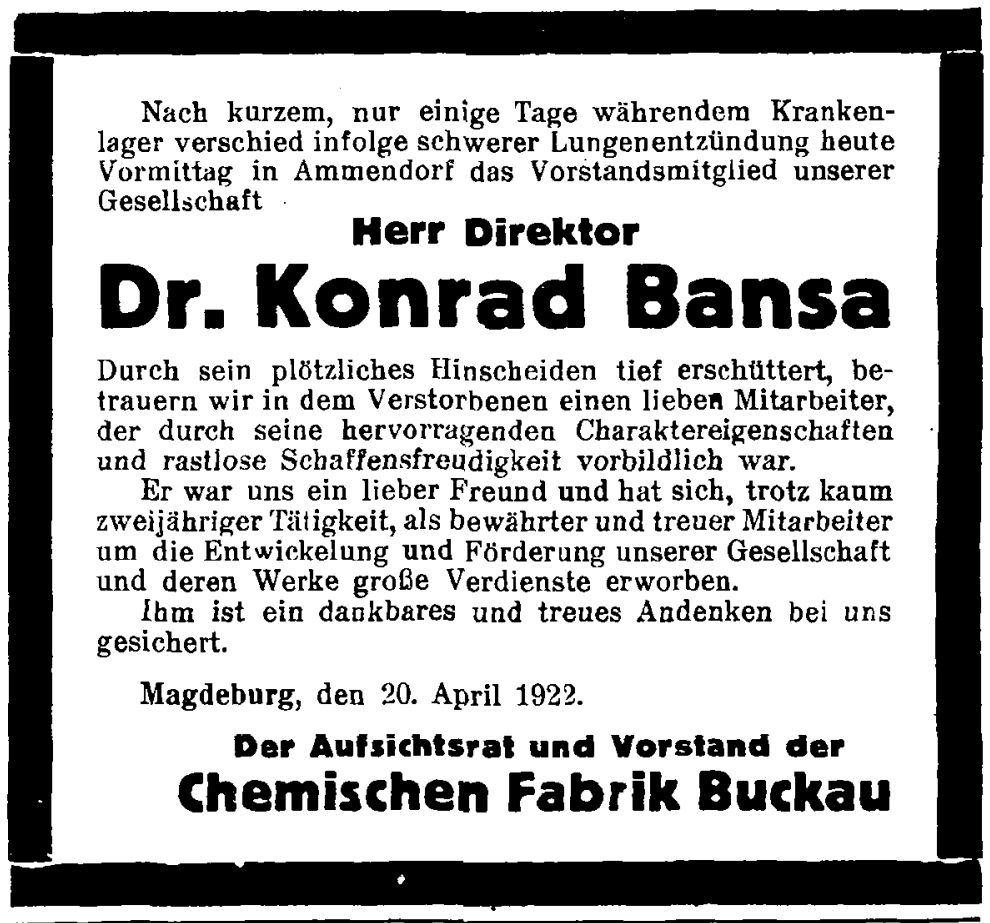

Nach kurzem, nur einige Tage währendem Krankenager verschied infolge schwerer Lungenentzündung heut Gesellschaft

\section{Dr. Konrad Bansa}

Durch sein plötzliches Hinscheiden tief erschuttert berauern wir in dem Verstorbenen einen lieben Mitarbeiter, and rastlose Schaffensfreudigkeit vorbildlich war.

Er war uns ein lieber Freund und hat sich, trotz kanm weijähriger Tätigkeit, als bewährter und treuer Mitarbeiter

Ihm ist ein dankbares und treues Andenken bei uns esichert.

\section{Der Aufichtsrat und Vorstand der Chemischen Fabrik Buckau}

